\title{
Field-induced motion of ferrofluid droplets through immiscible viscous media
}

\author{
S. AFKHAMI $I^{1}, Y$. RENARDY ${ }^{1}, M . R E N A R D Y^{1}$, \\ J. S. RIFFLE ${ }^{2}$ AND T. ST PIERRE \\ ${ }^{1}$ Department of Mathematics, Virginia Tech, Blacksburg, VA 24061-0123, USA \\ ${ }^{2}$ Department of Chemistry, Virginia Tech, Blacksburg, VA 24061-0212, USA \\ ${ }^{3}$ School of Physics, M013, The University of Western Australia, Crawley, WA 6009, Australia
}

(Received 19 February 2008 and in revised form 20 May 2008)

The motion of a hydrophobic ferrofluid droplet placed in a viscous medium and driven by an externally applied magnetic field is investigated numerically in an axisymmetric geometry. Initially, the drop is spherical and placed at a distance away from the magnet. The governing equations are the Maxwell equations for a non-conducting flow, momentum equation and incompressibility. A numerical algorithm is derived to model the interface between a magnetized fluid and a non-magnetic fluid via a volume-of-fluid framework. A continuum-surface-force formulation is used to model the interfacial tension force as a body force, and the placement of the liquids is tracked by a volume fraction function. Three cases are studied. First, where inertia is dominant, the magnetic Laplace number is varied while the Laplace number is fixed. Secondly, where inertial effects are negligible, the Laplace number is varied while the magnetic Laplace number is fixed. In the third case, the magnetic Bond number and inertial effects are both small, and the magnetic force is of the order of the viscous drag force. The time taken by the droplet to travel through the medium and the deformations in the drop are investigated and compared with a previous experimental study and accompanying simpler model. The transit times are found to compare more favourably than with the simpler model.

\section{Introduction}

Ferrofluids consist of magnetic nanoparticles in a colloidal solution. Recent developments in the synthesis and characterization of ferrofluids are motivated by biomedical applications (Liu et al. 2007), where the treatment of retinal detachment is one example (Mefford et al. 2007). A small amount of ferrofluid is injected into the vitreous cavity of the eye and guided by a permanent magnet inserted outside the scleral wall of the eye. The drop travels toward the side of the eye, until it can seal a retinal hole. The time taken for the drop to migrate is an important quantity which needs to be predicted, and which must be relatively short for the feasibility of this procedure. A simplified experimental model of this complex system is investigated in Mefford et al. (2007) with a ferrofluid drop, assumed to be a solid sphere, which moves through a highly viscous Newtonian fluid that represents the vitreous material (Nickerson et al. 2005). By treating the sphere as a magnetic particle, the magnetic force acting on it can be simplified as $F_{M}(x)=V M(x) \mu_{0}(\mathrm{~d} H / \mathrm{d} x)$, where $V$ is the volume of the sphere, $M$ is the magnetization of the ferrofluid droplet, $\mu_{0}$ is the permeability of vacumm, and $\mathrm{d} H / \mathrm{d} x$ is the gradient of the magnetic field $H$ with 
respect to the distance $x$ from the permanent magnet. This magnetic force is balanced with the viscous drag force on the sphere in Stokes flow given by $6 \pi \eta R_{0} U(x)$, to find the expression for $U(x)$. Integrating this over the distance to the magnet, an approximate time of travel was obtained and then compared with experiments conducted with a sphere filled with a liquid of viscosity $50 \mathrm{~Pa}$ s (sodium hyaluronate Provisc $^{\mathrm{TM}}$ solution commonly used in eye surgery). Their theoretical value was found to exceed the experimentally measured transit time by roughly $50 \%$. The authors noted one phenomenon in their experiments which was not included in their theory: at larger drop sizes, the shape deformed from a sphere to a teardrop as it accelerated toward the magnet. Separation of the tail of the teardrop was sometimes observed, resulting in smaller droplets that take longer to travel to the magnet. Another aspect of their estimate for the transit time is the use of the drag coefficient for a solid sphere rather than the viscosity-dependent value for a liquid sphere (Clift, Grace \& Weber 1978). The latter improves the gap between theory and experimental data, but still leaves significant discrepancies owing to drop deformation and coupled motion inside the drop.

The understanding of the above process is important for the efficient manipulation of the procedure. For instance, the size and the shape of the ferrofluid droplet can influence the motion of the droplet as it travels in a viscous medium. To investigate the response of a ferrofluid droplet to an applied magnetic field or to the capillary effects requires a thorough understanding of ferrohydrodynamics in such a system. The mathematical formulation of the flow of a ferrofluid is described by Rosensweig (1985). In this paper, we present a methodology for the numerical modelling of a two-phase system of immiscible fluids, a ferrofluid and a non-magnetic viscous medium. The magnetic force competes with the interfacial tension force and viscous drag to deform the drop. Previous numerical studies are limited to equilibrium shapes of ferrofluid drops (Lavrova et al. 2004, 2006) and interface instabilities (Bashtovoi et al. 2002; Matthies \& Tobiska 2005; Knieling et al. 2007). In all these studies, a finite-element method was used in which the governing equations of the magnetic liquid are coupled by the force balance at the interface and the surface tension is applied as a boundary condition at the interface. Here, we develop a numerical model, described in $\S 3$, and simulate the field-induced motion of a ferrofluid droplet in a viscous medium, with results presented in $\S 4$.

In this paper, the drop is assumed axisymmetric and deformable. We assume the drop size is small compared with the distance to the boundary of the eye. The magnetic field that is measured in the absence of the drop is used to generate boundary conditions. We investigate the transit time and drop shapes for a number of conditions that include those of Mefford et al. (2007).

\section{Governing equations}

A ferrofluid drop is suspended in a viscous medium that is non-magnetizable, as shown in figure 1. We assume that upon the placement of the magnet, the drop is instantly magnetized. The classical equations for the evolution of the two-fluid system are the Maxwell equations, the incompressible Navier-Stokes equations, and a constitutive relationship for the magnetic induction $\boldsymbol{B}(\mathrm{T})$, magnetic field $\boldsymbol{H}\left(\mathrm{A} \mathrm{m}^{-1}\right)$, and magnetization $\boldsymbol{M}\left(\mathrm{A} \mathrm{m}^{-1}\right)$ (Lavrova et al. 2006). In SI units, $\boldsymbol{M}=\chi_{m} \boldsymbol{H}$ and

$$
\boldsymbol{B}(\boldsymbol{x}, t)= \begin{cases}\mu_{1} \boldsymbol{H} & \text { in the ferrofluid, } \\ \mu_{0} \boldsymbol{H} & \text { in the viscous medium, }\end{cases}
$$




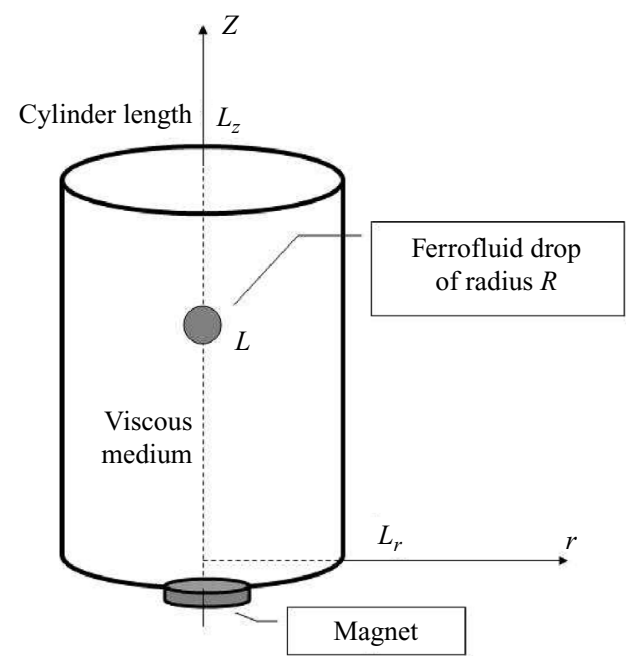

FiguRE 1. Schematic of the initial configuration. The computational box covers $0 \leqslant z \leqslant L_{z}$, $0 \leqslant r \leqslant L_{r}$. Initially, a spherical ferromagnetic drop of radius $R$ is placed a distance $L$ from the magnet at $(r, z)=(0,0)$.

where the magnetic permeability of the ferrofluid is $\mu_{1}=\mu_{0}\left(1+\chi_{m}\right)$, and $\chi_{m}$ is its magnetic susceptibility. $\mu_{0}=4 \pi \times 10^{-7} \mathrm{~N} \mathrm{~A}^{-2}$ is the permeability of vacuum, as well as many other non-ferromagnetic materials. The Maxwell equations for a nonconducting fluid are $\nabla \cdot \boldsymbol{B}=0$ and $\nabla \times \boldsymbol{H}=0$. The latter yields a magnetic scalar potential $\psi$, where $\boldsymbol{H}=\nabla \psi$. The former yields

$$
\nabla \cdot(\mu \nabla \psi)=0
$$

The permeability is a constant per fluid (except as indicated in $\S 4.3$ ), and jumps in value across the interface, so that $\psi(\boldsymbol{x}, t)$ changes as the interface evolves.

The boundary condition on the magnetic field is reconstructed from the experimental measurements of Mefford et al. (2007). In the absence of the drop, they measured the magnitude $H(z)$ as a function of distance from the magnet, $z$, and fitted the data to a fifth-degree polynomial, as shown in figure 2. The scalar potential is then a sixth-degree polynomial $\phi(0, z)=P_{6}(z)$ along the axis of the cylindrical domain. In the absence of the drop, $\phi$ satisfies Laplace's equation

$$
\frac{1}{r} \frac{\partial}{\partial r}\left(r \frac{\partial \phi}{\partial r}\right)+\frac{\partial^{2} \phi}{\partial z^{2}}=0 .
$$

If there is a solution, it is analytic and has $r^{2}$-symmetry. The ansatz $\phi(r, z)=P_{6}(z)+$ $r^{2} P_{4}(z)+r^{4} P_{2}(z)+r^{6} P_{0}(z)$ yields

$$
\phi(r, z)=P_{6}(z)-\frac{1}{4} r^{2} P_{6}^{\prime \prime}(z)+\frac{1}{64} r^{4} P_{6}^{(\mathrm{iv})}(z)-\frac{1}{(36)(64)} r^{6} P_{6}^{(\mathrm{vi})}(z) .
$$

This yields the boundary condition, and also approximates an initial condition when the drop is relatively small. The lateral size of the computational domain is chosen to be sufficiently large so that it is consistent with the assumption that results do not change if a larger size were used (see $\S 4.1)$. These checks were done by calculating the solution for double the lateral domain size. 


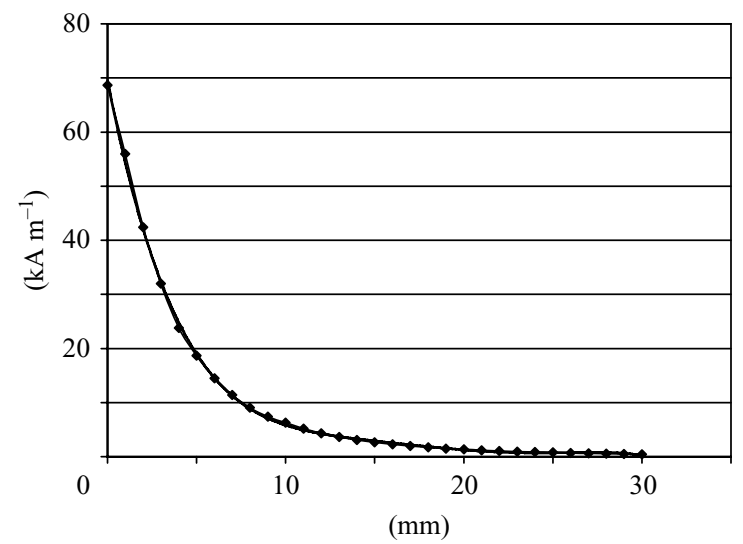

Figure 2. Measured data for the magnetic field from figure 3 of Mefford et al. (2007) ( $\bullet$ ) and fifth-degree polynomial fitted to the data (-) as functions of the distance from the magnet. $y=-\left(3 \times 10^{-5}\right) x^{5}+0.0025 x^{4}-0.0954 x^{3}+1.7906 x^{2}-16.99 x+69.605$.

The magnetic potential $\psi$ is calculated from (2.2). In axisymmetric cylindrical coordinates,

$$
\frac{1}{r} \frac{\partial}{\partial r}\left(\mu r \frac{\partial \psi}{\partial r}\right)+\frac{\partial}{\partial z}\left(\mu \frac{\partial \psi}{\partial z}\right)=0 \quad \text { in } \Omega .
$$

where $\Omega$ denotes the computational domain. The boundary conditions for $\psi$ on the domain boundaries $\partial \Omega$ are defined as

$$
\frac{\partial \psi}{\partial n}=\frac{\partial \phi}{\partial n} \quad \text { on } \quad \partial \Omega
$$

where $\partial / \partial n=\boldsymbol{n} \cdot \nabla$, and $\boldsymbol{n}$ denotes the normal to the boundary $\partial \Omega$.

In order to impose the boundary condition in our numerical model, we perform a transformation of variables to $\zeta: \psi=\phi+\zeta$, where $\phi$ is the potential field without the magnetic medium. We can then rewrite (2.2) such that

$$
\nabla \cdot(\mu \nabla \zeta)=-\nabla \cdot(\mu \nabla \phi),
$$

where $\nabla \cdot(\mu \nabla \phi)$ vanishes everywhere except on the surface between the drop and the surrounding fluid $\partial \Omega_{f}$ and

$$
\frac{\partial \zeta}{\partial n}=0 \quad \text { on } \quad \partial \Omega
$$

The well-known Langevin function $L(\alpha)=\operatorname{coth} \alpha-\alpha^{-1}$ is used to describe the magnetization $M=|\boldsymbol{M}|$ behaviour of the ferrofluid versus the strength of the magnetic field $\boldsymbol{H}$ :

$$
\boldsymbol{M}(\boldsymbol{H})=M_{s} L\left(\frac{\mu_{0} m|\boldsymbol{H}|}{k_{B} T}\right) \frac{\boldsymbol{H}}{|\boldsymbol{H}|},
$$

where the saturation magnetization $M_{s}$ and the magnetic moment of the particle enter as parameters, $T$ denotes the temperature, and $k_{B}$ is the Boltzmann's constant. Figure 3 compares $\boldsymbol{M}$ vs. $\boldsymbol{H}$ for the measured data of Mefford et al. (2007) and the Langevin fit. It is evident that the Langevin function fits the respective experimental data reasonably well. 


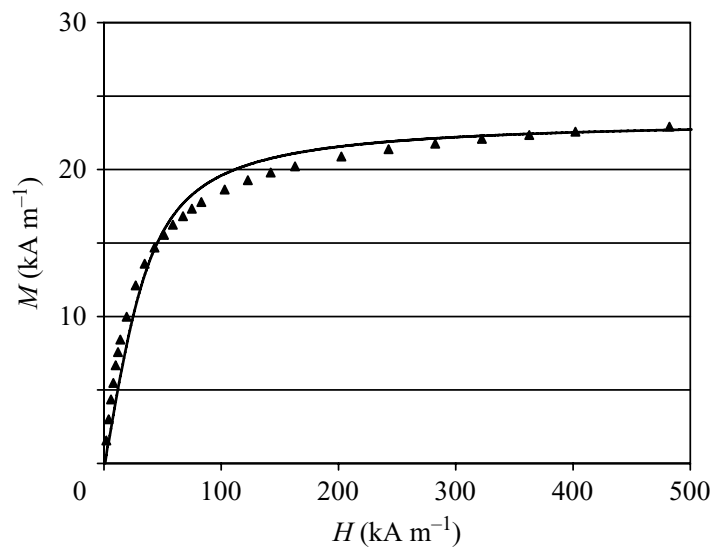

Figure 3. Magnetization behaviour of the ferrofluid containing 7 vol. $\%$ of magnetite $\left(\mathrm{Fe}_{3} \mathrm{O}_{4}\right)$ particles with a mean diameter of $7 \mathrm{~nm}$ for figure 3 of Mefford et al. (2007). Measured data $(\Delta)$ are compared with the Langevin fit $(-)$, assuming each of the magnetite particles has a total magnetic moment $\approx 2 \times 10^{-19} \mathrm{~A} \mathrm{~m}^{2}$.

Each liquid is identified with a colour function,

$$
C(r, z, t)= \begin{cases}0 & \text { in the viscous medium, } \\ 1 & \text { in the ferrofluid drop, }\end{cases}
$$

which advects with the flow. The position of the interface is given by the discontinuities in the colour function. The fluid equation of motion is

$$
\rho \frac{\mathrm{d} \boldsymbol{u}}{\mathrm{d} t}=-\nabla p+\nabla \cdot \eta \boldsymbol{S}+\boldsymbol{F}_{s}+\nabla \cdot \boldsymbol{\sigma}_{m}, \quad S_{i, j}=\frac{1}{2}\left(\frac{\partial u_{j}}{\partial x_{i}}+\frac{\partial u_{i}}{\partial x_{j}}\right),
$$

where $\boldsymbol{F}_{s}$ denotes the continuum body force due to interfacial tension,

$$
\boldsymbol{F}_{s}=\gamma \tilde{\kappa} \boldsymbol{n} \boldsymbol{\delta}_{S}, \quad \tilde{\kappa}=-\nabla \cdot \boldsymbol{n} .
$$

$\gamma$ denotes the coefficient of interfacial tension, $\boldsymbol{n}=\nabla C /|\nabla C|$ is the normal to the interface, $\delta_{S}=|\nabla C|$ is the delta-function at the interface, and $\tilde{\kappa}$ is the curvature. The viscous stress tensor is $\eta S_{i, j}$ where the rate of deformation tensor is $S_{i, j}$. The magnetic stress tensor $\boldsymbol{\sigma}_{m}$ is derived in the Appendix to be $\boldsymbol{B} \boldsymbol{H}^{T}$, so that the equation of motion becomes

$$
\rho \frac{\mathrm{d} \boldsymbol{u}}{\mathrm{d} t}=-\nabla p+\nabla \cdot \eta \boldsymbol{S}+\boldsymbol{F}_{s}+\nabla \cdot \boldsymbol{B} \boldsymbol{H}^{T},
$$

to be interpreted as a weak formulation.

\section{Numerical methodology}

In the absence of an initially imposed velocity and gravity, and using the following normalizations for a drop of initial radius $R_{0}$,

$$
\begin{gathered}
\boldsymbol{x}^{*}=\boldsymbol{x} / R_{0}, t^{*}=t \eta_{0} /\left(\rho_{0} R_{0}^{2}\right), \eta^{*}=\eta / \eta_{0}, \rho^{*}=\rho / \rho_{0}, \\
\boldsymbol{u}^{*}=\boldsymbol{u} \rho_{0} R_{0} / \eta_{0}, \quad p^{*}=p \rho_{0} R_{0}^{2} /\left(\eta_{0}\right)^{2}, \boldsymbol{H}^{*}=\boldsymbol{H} / H_{0},
\end{gathered}
$$


the equation of motion becomes

$$
\rho^{*} \frac{\mathrm{d} \boldsymbol{u}^{*}}{\mathrm{~d} t^{*}}=-\nabla^{*} p^{*}+\nabla^{*} \cdot \eta^{*} \boldsymbol{S}^{*}+L a \boldsymbol{F}_{s}^{*}+L a_{m} \nabla^{*} \cdot \boldsymbol{\sigma}_{m}^{*}
$$

where the subscript 0 refers to the droplet; i.e. $\rho_{0}$ and $\eta_{0}$ are the ferrofluid density and viscosity, respectively, and $H_{0}$ is the characteristic scale of the magnetic field strength. The Laplace number,

$$
L a=\gamma \rho_{0} R_{0} / \eta_{0}^{2}
$$

is the ratio of the surface tension to the viscous drag (note that $L a=1 /(O h)^{2}$ where $O h$ is the Ohnesorge number). The magnetic Laplace number (or magnetic Reynolds number),

$$
L a_{m}=\mu_{0} H_{0}^{2} \rho_{0} R_{0}^{2} / \eta_{0}^{2},
$$

is the ratio of the magnetic force to inertial force. The ratio of magnetic force to interfacial tension force is named the magnetic Bond number (Baygents, Rivette \& Stone 1998; Voltairas, Fotiadis \& Michalis 2002),

$$
B o_{m}=L a_{m} / L a \text {. }
$$

A volume-of-fluid algorithm on a marker-and-cell (MAC) grid of equidistant mesh $\Delta$ and a computational domain $L_{r} \times L_{z}$ is used. The discretized colour function gives the volume fraction of the ferrofluid. The advection of the volume fraction function is Lagrangian, and the piecewise linear interface reconstruction scheme (PLIC) is used to calculate the interface position at each time step. The details of the method for the Navier-Stokes equations are given in Lafaurie et al. (1994), Li \& Renardy (1999), Scardovelli \& Zaleski (1999) and Li, Renardy \& Renardy (2000) and not repeated here. Briefly, a provisional velocity field is first predicted and then corrected with the pressure field that is calculated as a solution of a Poisson problem. Interfacial tension is discretized using the continuum-surface-force model (Brackbill, Kothe \& Zemach 1992). The new aspect is the extension of the algorithm to the ferrofluid.

The magnetic potential field is discretized using second-order central differences and is computed as a solution of the Poisson problem (2.6). In axisymmetric coordinates, the discretization of $(2.6)$ at cell $(i, j)$ yields

$$
\begin{aligned}
\nabla \cdot(\mu \nabla \zeta)_{i, j}= & \frac{1}{r_{i, j}}\left(r_{i+1 / 2, j} \mu_{i+1 / 2, j}\left(\frac{\partial \zeta}{\partial r}\right)_{i+1 / 2, j}-r_{i-1 / 2, j} \mu_{i-1 / 2, j}\left(\frac{\partial \zeta}{\partial r}\right)_{i-1 / 2, j}\right) / \Delta \\
& +\left(\mu_{i, j+1 / 2}\left(\frac{\partial \zeta}{\partial z}\right)_{i, j+1 / 2}-\mu_{i, j-1 / 2}\left(\frac{\partial \zeta}{\partial z}\right)_{i, j-1 / 2}\right) / \Delta
\end{aligned}
$$

where, for instance for the cell face $(i+1 / 2, j)$,

$$
\left(\frac{\partial \zeta}{\partial r}\right)_{i+1 / 2, j}=\frac{\zeta_{i+1, j}-\zeta_{i, j}}{\Delta}
$$

A weighted harmonic mean interpolation is used to compute $\mu$ at cell face $(i+1 / 2, j)$ :

$$
\frac{1}{\mu_{i+1 / 2, j}}=\frac{1}{2}\left(\frac{1}{\mu_{i, j}}+\frac{1}{\mu_{i+1, j}}\right),
$$




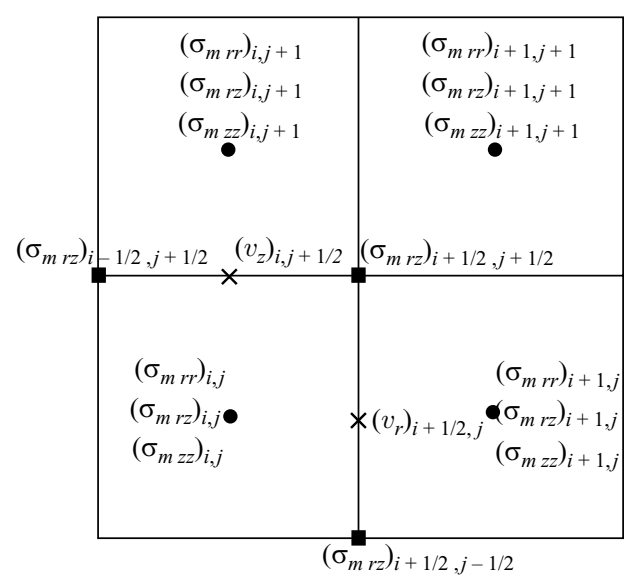

FIGURE 4. Location of the velocities and the magnetic stress tensor components on a MAC grid. Corner values of the magnetic stress tensor components $(r z$, at $\mathbf{\square})$ are calculated from cell-centre values $(\bullet)$.

where

$$
\frac{1}{\mu_{i, j}}=\frac{1-C_{i, j}}{\mu_{0}}+\frac{C_{i, j}}{\mu_{1}},
$$

and the discretized colour function $C_{i j}$ represents the volume fraction of the ferrofluid in cell $(i, j)$ (Patankar 1980). Analogous relationships can be written for other faces of a cell. The right-hand side of (2.6) is discretized similarly. The boundary condition for cells on the solid boundary is a second-order discretization of a zero gradient boundary condition for $\zeta: \partial \zeta / \partial n=0$. A multigrid Poisson solver is then used to obtain the solution of the resulting linear set of equations.

The spatial discretization of the velocity field is based on the MAC grid in figure 4. Therefore, the evaluation of the components of the magnetic stress tensor requires the evaluation of gradients at faces. In axisymmetric coordinates, the divergence of the magnetic stress tensor is discretized as

$$
\begin{aligned}
\boldsymbol{e}_{r} & : \frac{1}{r_{i+1 / 2, j}} \frac{r_{i+1, j}\left(\left(\sigma_{m}\right)_{r r}\right)_{i+1, j}-r_{i, j}\left(\left(\sigma_{m}\right)_{r r}\right)_{i, j}}{\Delta} \\
& +\frac{\left(\left(\boldsymbol{\sigma}_{m}\right)_{r z}\right)_{i+1 / 2, j+1 / 2}-\left(\left(\boldsymbol{\sigma}_{m}\right)_{r z}\right)_{i+1 / 2, j-1 / 2}}{\Delta} \\
\boldsymbol{e}_{z}: & \frac{1}{r_{i, j+1 / 2}} \frac{r_{i+1 / 2, j+1 / 2}\left(\left(\boldsymbol{\sigma}_{m}\right)_{r z}\right)_{i+1 / 2, j+1 / 2}-r_{i-1 / 2, j+1 / 2}\left(\left(\sigma_{m}\right)_{r z}\right)_{i-1 / 2, j+1 / 2}}{\Delta} \\
& +\frac{\left(\left(\boldsymbol{\sigma}_{m}\right)_{z z}\right)_{i, j+1}-\left(\left(\boldsymbol{\sigma}_{m}\right)_{z z}\right)_{i, j}}{\Delta},
\end{aligned}
$$

where the components such as $\left(\sigma_{m}\right)_{r r}$ are defined in the Appendix. Second-order central differences are used to discretize the components of the magnetic stress tensor at the centre of a cell and a simple averaging from cell-centre values is used to extrapolate the magnetic stress components to cell corners.

\section{Results}

Numerical simulations are presented in three parts. Section 4.1 concerns tests of the numerical implementation by focusing on the resulting magnetic fields and by testing 


\begin{tabular}{|c|c|c|c|}
\hline Section & $\S 4.2$ & $\S 4.2$ & $\S 4.3$ \\
\hline $2 R_{0}(\mathrm{~mm})$ & 2.5 & 1 & $1,1.8,2$ \\
\hline$L_{r}$ & $1.6 R_{0}$ & $4 R_{0}$ & $4 \mathrm{~mm}$ \\
\hline$L_{z}$ & $12.8 R_{0}$ & $24 R_{0}$ & $16 \mathrm{~mm}$ \\
\hline$\Delta$ & $R_{0} / 20$ & $R_{0} / 12$ & $R_{0} / 8, R_{0} / 9, R_{0} / 10$ \\
\hline$\rho_{0}$ & $1.32 \rho_{v}$ & $1.32 \rho_{v}$ & $1320 \mathrm{~kg} \mathrm{~m}^{-3}$ \\
\hline$\eta_{0}$ & $1.5 \eta_{v}$ & $1.5 \eta_{v}$ & $80 \mathrm{~kg} \mathrm{~m}^{-1} \mathrm{~s}^{-1}$ \\
\hline$\rho_{v}$ & & & $998 \mathrm{~kg} \mathrm{~m}^{-3}$ \\
\hline$\eta_{v}$ & & & $50 \mathrm{~kg} \mathrm{~m}^{-1} \mathrm{~s}^{-1}$ \\
\hline$x_{m}$ & 0.25 & 0.25 & * \\
\hline$H_{0}\left(\mathrm{kA} \mathrm{m}^{-1}\right)$ & 1 & 1 & 1 \\
\hline $\mathrm{La}$ & 5.15 & $0.002,0.01,0.04,0.1,0.4$ & \\
\hline$L a_{m}$ & $0.3,1.5,3.6$ & 0.05 & \\
\hline$B o_{m}$ & $0.06,0.3,0.6,1.2$ & $24,5,1,0.5,0.1$ & $0.031,0.056,0.063$ \\
\hline
\end{tabular}

TABLE 1. Overview of the sets of simulations presented in $\S \S 4.2-4.3$.

for convergence of the solution with grid refinement. Section 4.2 presents a parametric study, varying $L a_{m}$ for fixed $L a$, and vice versa. Section 4.3 contains the application of our model to the experimental data of Mefford et al. (2007), and examines the time taken by the drop to reach the magnet.

Table 1 provides a comprehensive overview of the sets of simulations presented in the remainder of this section. For $\S 4.3$, the magnetic susceptibility is computed using the Langevin function via (2.8), and this yields improved agreement with experimental data over the linear variation defined by a constant $\chi_{m}$. The characteristic scale of the magnetic field strength $H_{0}$ is taken to be $1 \mathrm{kA} \mathrm{m}^{-1}$ in all the cases in $\S 4.2$, since this is of the same order as the magnetic field strength which is initially inducted by the magnet on the droplet placed about $12 \mathrm{~mm}$ away from the permanent magnet (cf. figure 2). Note that the magnetic field strength varies with location and the choice of a characteristic scale is not straightforward. Thus, as the droplet moves toward the magnet, the effective magnetic Laplace number increases well beyond our nominal value.

\subsection{Magnetic field and imposed boundary condition}

Figure 5 shows a convergence test for the calculated travel times, at different mesh sizes in units of the initial droplet radius $R_{0}$. A droplet of radius $1 \mathrm{~mm}$ is centred at a distance $10 \mathrm{~mm}$ away from the bottom of the $16 \mathrm{~mm} \times 4 \mathrm{~mm}$ domain. The time that is required for the droplet to reach the bottom of the domain is calculated at different mesh sizes to demonstrate the spatial convergence of the numerical results. The magnetic susceptibility used in this case is considered to be constant and $\chi_{m}=0.25$.

We demonstrate the effectiveness of our methodology by presenting the results of the simulated applied magnetic field using the magnetic field boundary condition (2.3), compared with values measured along the centreline of the domain by Mefford et al. (2007). Figure 6 shows the computed magnetic field along the centreline of the $16 \mathrm{~mm} \times 4 \mathrm{~mm}$ domain compared with the measured magnetic field generated by a permanent magnet in the absence of a droplet. The agreement is excellent, and this also provides a check that the lateral boundary of the computational domain is sufficiently far away from the drop. 


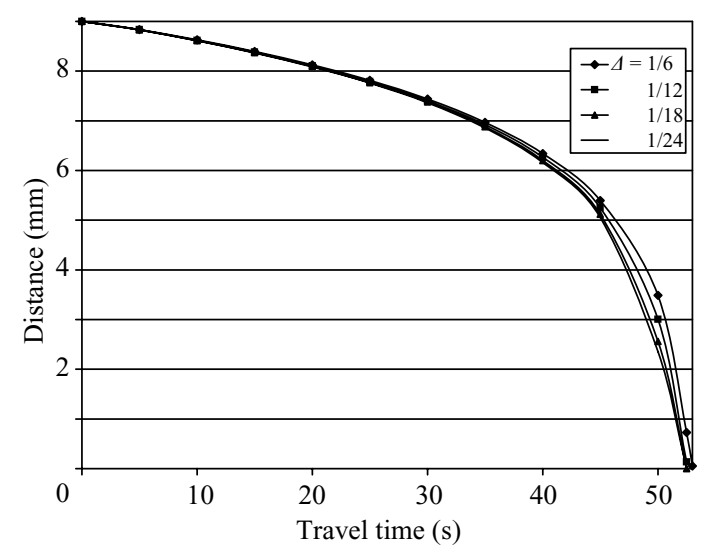

FIgURE 5. Calculated transit times at different mesh sizes in units of initial drop radius $R_{0}$. Results are shown to be convergent as the mesh is refined. The parameters are those of $\S 4.3$.

In figure 6, the computed magnetic field in the presence of a $2 \mathrm{~mm}$ diameter droplet centred at distances $12 \mathrm{~mm}, 8 \mathrm{~mm}$ and $4 \mathrm{~mm}$ from the bottom of the computational domain are also presented. Comparison of the variation of numerical results of the magnetic field across the interface is used to check the necessary continuity of $\boldsymbol{B} \cdot \boldsymbol{n}$.

In figure 7, the magnetic field lines and contour plots of the magnetic field amplitude are plotted for cases of a $2 \mathrm{~mm}$ diameter droplet centred at distances $12 \mathrm{~mm}, 8 \mathrm{~mm}$ and $4 \mathrm{~mm}$ from the bottom of the computational domain. The magnetic field lines in the viscous medium that is non-magnetizable are distorted in the presence of the ferrofluid droplet because of having different permeability.

\subsection{Variation with La and $L a_{m}$}

Past theoretical studies have shown that microscopic ferrofluid droplets $(2-20 \mu \mathrm{m})$ deform to prolate droplets in the direction of the uniform applied magnetic field (Bacri \& Salin 1982). Here we also numerically observe that drops elongate in the presence of non-uniform magnetic fields. The computational domain is $1.6 R_{0} \times 12.8 R_{0}$. A freely suspended ferrofluid droplet of radius $R_{0}(1.25 \mathrm{~mm})$ is initially centred at $(0$, $\left.10.4 R_{0}\right)$. The permanent magnet is at the bottom of the domain. At the walls, the velocities satisfy no slip. Because of symmetry, only half of the domain is simulated. The mesh size is $\Delta=R_{0} / 20$.

The results of the ferrofluid drop elongation upon the magnetic Laplace number $L a_{m}$ are presented. The value of the magnetic susceptibility is $\chi_{m}=0.25$ and chosen to be constant during the process. The density ratio is $\rho_{\text {droplet }} / \rho_{\text {viscous }}=1.32$ and the viscosity ratio is $\eta_{\text {droplet }} / \eta_{\text {surrounding }}=1.5$. The Laplace number is $L a=5.15$. Figure 8 shows droplet shapes for magnetic Laplace numbers $L a_{m}=0.3,1.5,3$ and 6 at nondimensional times $\tau=t \eta_{0} /\left(\rho_{0} R_{0}^{2}\right)$. These figures show that the increase of the magnetic field results in a drop elongation in the direction of the applied magnetic field. While for $L a_{m}=0.3$ the shape of the droplet remains almost round for all time (figure $8 a$ ), higher magnetic Laplace numbers result in a dramatic deviation from round shapes to further elongated shapes forming columnar configurations. Figure 8 shows that increase of the magnetic Laplace number $L a_{m}$ results in a continuous drop prolation accompanied by a deformation from a round shape to a tear-drop shape. At the highest magnetic Laplace number (figure $8 d$ ), small surface undulations begin to appear on the flat sides of the front of the droplet. 

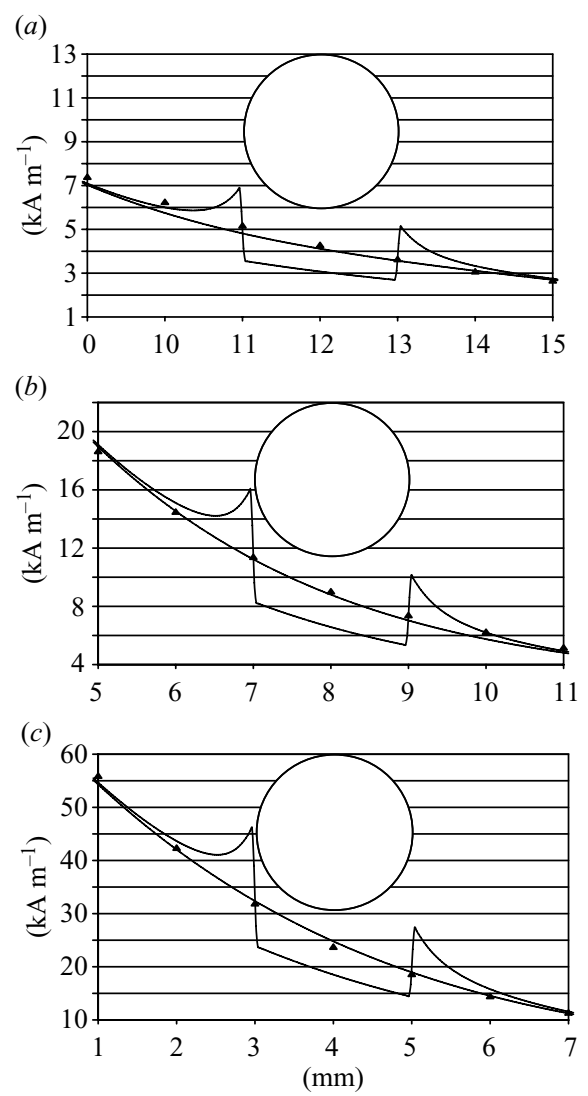

FIGURE 6. Distribution of the magnetic field $\left(\mathrm{kA} \mathrm{m}^{-1}\right)$ along the centreline of the computational domain. The computed magnetic field along the centreline of the domain in the absence of the drop (-) is compared with the measured magnetic field generated by a permanent magnet ( $\boldsymbol{\Delta}$ ) from Mefford et al. (2007). The computed magnetic field in the presence of a $2 \mathrm{~mm}$ droplet (-) is superposed when the droplet is placed at distances $(a) 12 \mathrm{~mm}$, (b) $8 \mathrm{~mm}$ and (c) $4 \mathrm{~mm}$ from the magnet, respectively. $\chi_{m}=1$.

Figure 9 depicts velocity fields at $\tau=560,15,3.3$ and 1 corresponding to $L a_{m}=0.3$, $1.5,3$ and 6 , respectively. The motion of the droplet is a function of the variation of the magnetic field within the droplet, i.e. the front of the droplet feels a higher magnetic force than the back of the droplet. This effect can be observed from velocity fields in figures $9(b)$ to $9(d)$ where the portion of the droplet closer to the magnet accelerates much faster towards the magnet rather than the section at the back of the droplet.

Ferrofluid drops with different interfacial tension energies deform differently under an applied magnetic field. A lower surface tension can result in the deviation from a round shape to a prolate ellipsoid structure which can consequently lead to a higher droplet velocity. A freely suspended ferrofluid droplet of radius $R_{0}(0.5 \mathrm{~mm})$ is initially centred at $\left(0,20 R_{0}\right)$. The permanent magnet is at the bottom of the domain. At the walls, the velocities satisfy no slip. Because of symmetry, only half of the domain is simulated. The mesh size is $\Delta=R_{0} / 12$, and the computational domain is $4 R_{0} \times 24 R_{0}$. Figure 10 plots the calculated transit times for Laplace numbers $L a=0.002,0.01$, $0.04,0.1$ and 0.4 for fixed $L a_{m}=0.05$. It is evident that the velocity of the droplet 

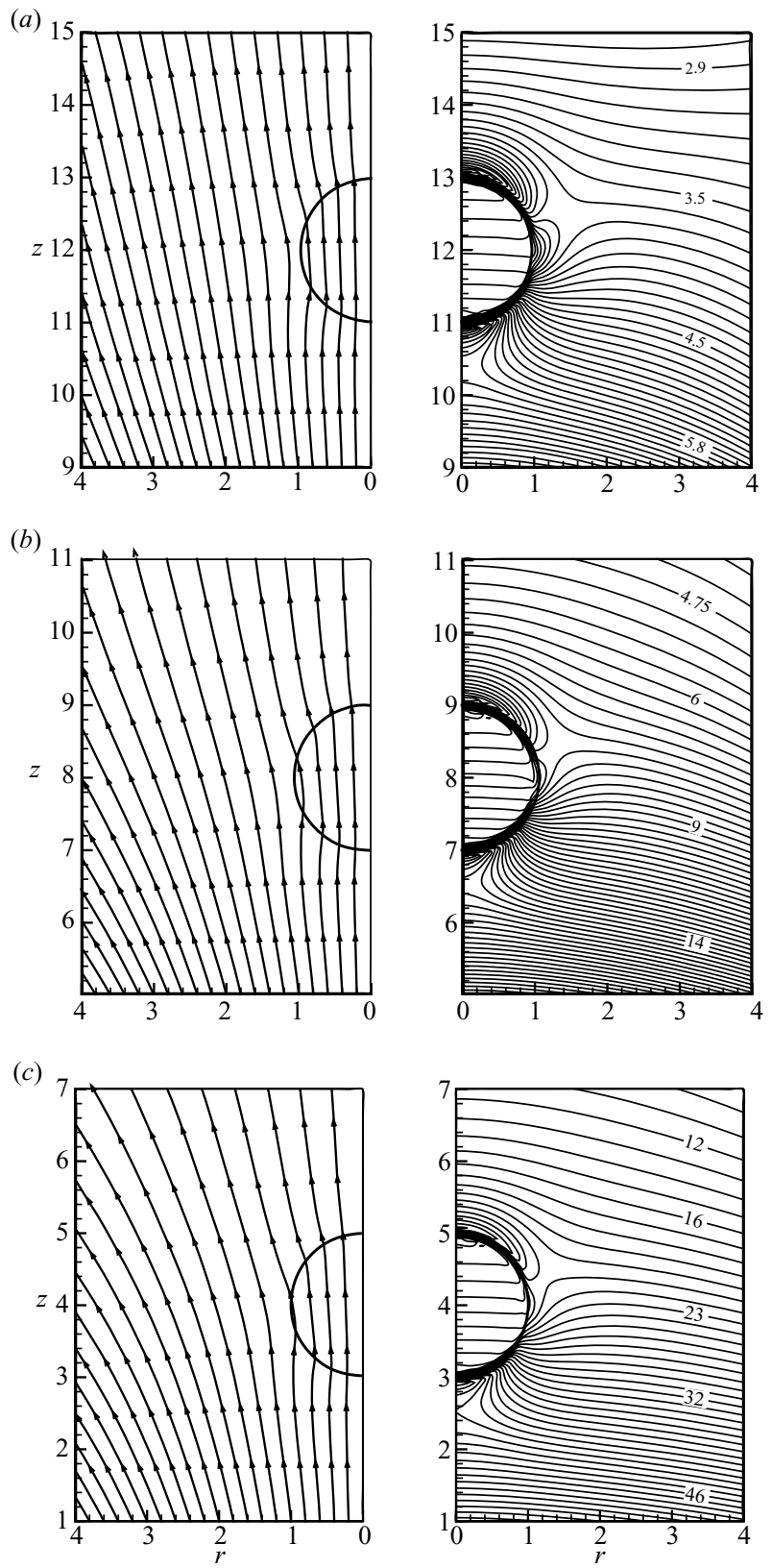

FIGURE 7. Magnetic field lines (left) and contours of the magnetic field amplitude $\left(\mathrm{kA} \mathrm{m}^{-1}\right.$, right) in the presence of a ferrofluid droplet in a non-magnetizable medium. A droplet of diameter $2 \mathrm{~mm}$ is centred at distances $(a) 12 \mathrm{~mm},(b) 8 \mathrm{~mm}$ and $(c) 4 \mathrm{~mm}$ above the bottom of the computational domain. $\chi_{m}=1$.

varies as a function of the Laplace number for low inertia. A lower surface tension alters the shape of the droplet which accounts for the variation in the velocity of the droplet.

In figure 11, the motion of ferrofluid droplets are shown at different Laplace numbers at non-dimensional times $\tau$. At low Laplace numbers, the round droplet 
(a)

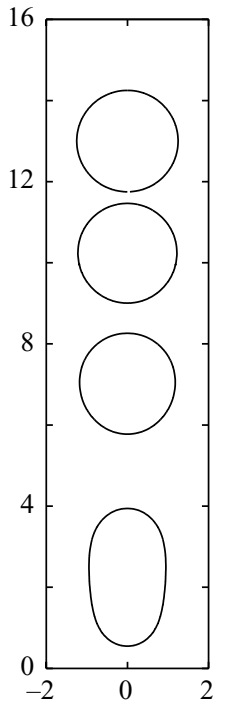

(b)

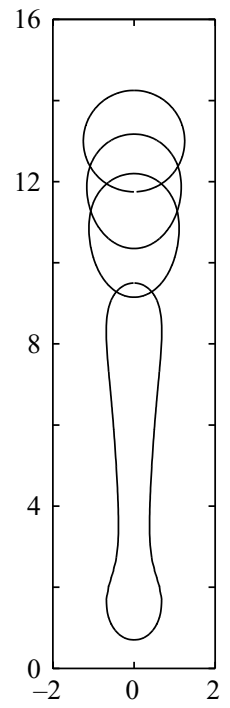

(c)

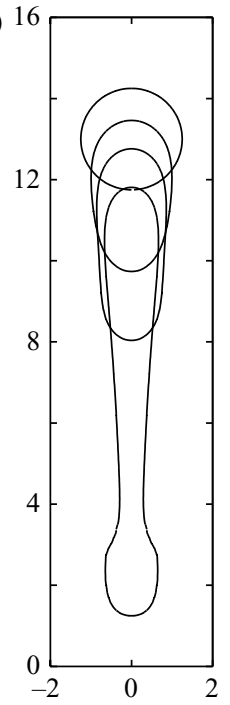

(d)

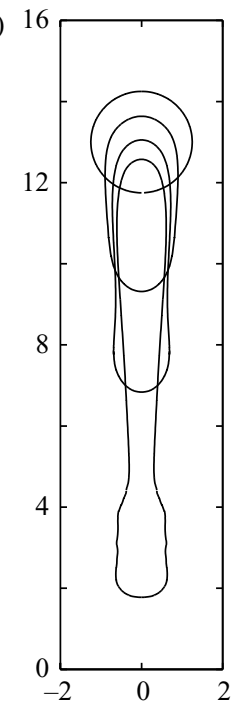

FIGURE 8. Droplet shapes at different magnetic Laplace numbers, at fixed $L a=5.15$ : (a) $L a_{m}=0.3, \tau=0,410,500,560 ;$ (b) $L a_{m}=1.5, \tau=0,7.5,11.2,15 ;$ (c) $L a_{m}=3, \tau=0,1.9$, 2.6, 3.3; (d) $L a_{m}=6, \tau=0,0.6,0.9,1$. The magnetic Bond numbers are $B o_{m}=(a) 0.06,(b) 0.3$, (c) $0.6,(d) 1.2$.

deforms in the direction of the applied magnetic field forming a prolate shape which in turn influences the motion of the droplet. In figures 8 and 9, the magnetic Bond number varies from 0.06 to 1.2 with increase in $L a_{m}$, and results in highly deformed drops for the higher $B o_{m}$. Similarly, though at lower inertia, figure 11 shows that as the Laplace number varies from 0.002 to $0.4, B o_{m}$ decreases from 24 to 0.1 , and drop deformation decreases. Hence, at both order 1 inertia and small inertia, the drop deforms more for higher $\mathrm{Bo}_{m}$, where the effect of magnetic force is more important than interfacial tension force.

When increasing the magnetic Laplace number, the differential between the force at the front of the drop to that at the rear becomes more pronounced and may become strong enough to overcome the tendency of surface tension to keep the drop round. Conversely, when keeping the magnetic Laplace number constant but varying the Laplace number, the ratio of the magnetic effect to the surface tension effect becomes more favourable as the Laplace number becomes small, and the fact that the strength of the magnetic force is differentially higher at the front becomes more important. Note that a comparison of figures 8 and 11 shows quite different drop shapes for comparable magnetic Bond numbers. Hence, the evolution of drop shapes is dependent on both the magnetic Laplace number and the Laplace number.

\subsection{Simulation for the parameters of Mefford et al. (2007)}

The simulation results of the magnetic field-induced motion of PDMS ferrofluid droplets in a viscous medium are presented. The diameter and initial position of the ferrofluid drop are varied. At the walls, the velocities satisfy no slip. Because of symmetry, only half of the domain is simulated.

A series of computations are performed to calculate the time taken by the ferrofluid droplet through the viscous medium until the magnet is reached. The droplet models a PDMS ferrofluid, with density $1320 \mathrm{~kg} \mathrm{~m}^{-3}$, and viscosity $80 \mathrm{Pas}$. The interfacial 
(a)

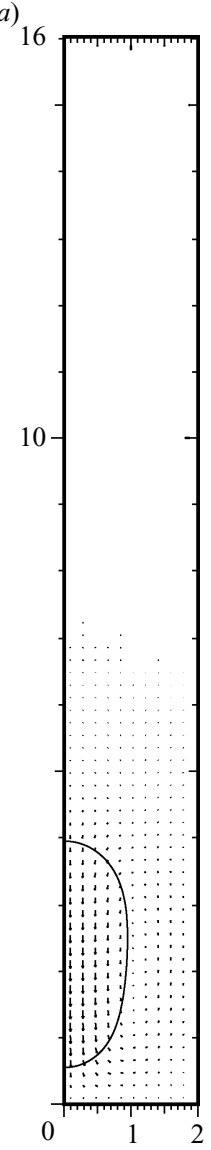

(b)

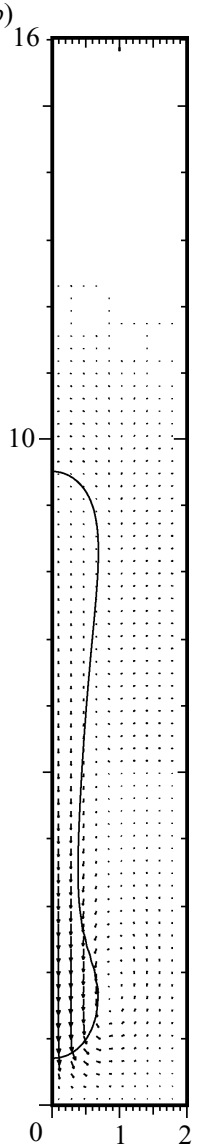

(c)

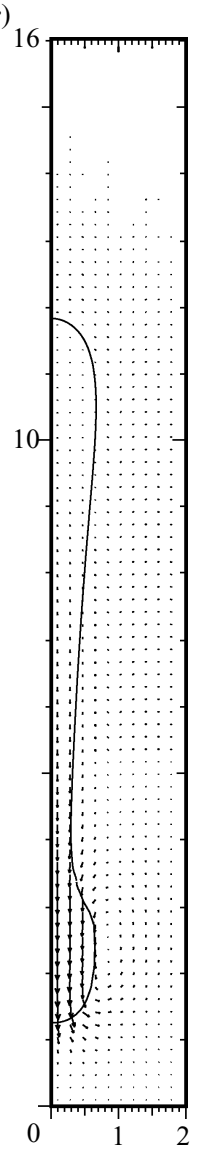

(d)

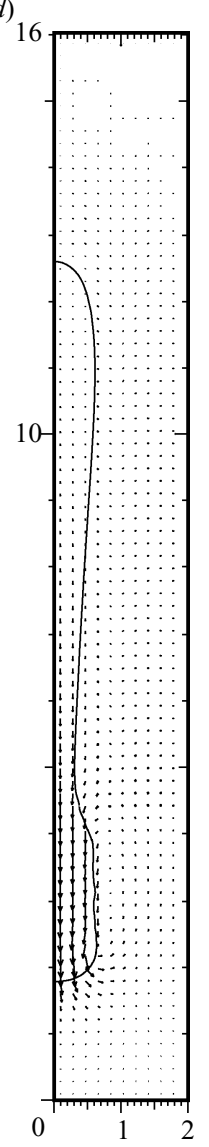

FIGURE 9. Velocity fields at different magnetic Laplace numbers with fixed $L a=5.15$ : $(a)$ $L a_{m}=0.3, \tau=325$, (b) $L a_{m}=1.5, \tau=15$, (c) $L a_{m}=3, \tau=3.3$, (d) $L a_{m}=6, \tau=1$. The magnetic Bond numbers are $B o_{m}=(a)$ 0.06, (b) 0.3, (c) 0.6, (d) 1.2.

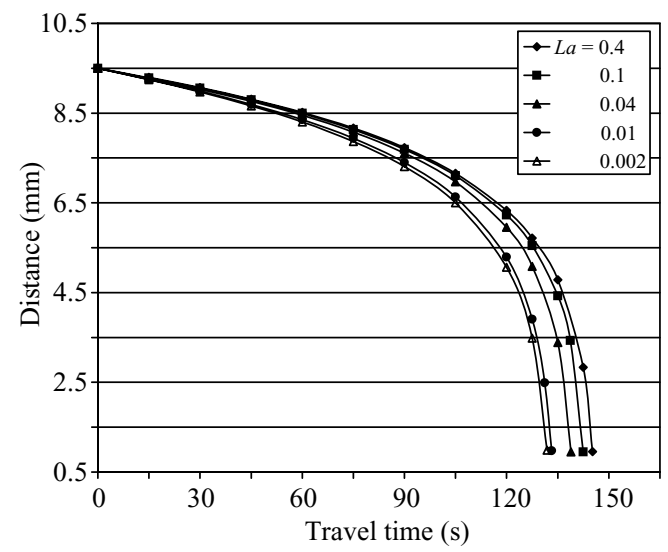

Figure 10. Calculated transit times at Laplace numbers ranging from 0.002 to 0.4 , corresponding to magnetic Bond numbers from 24 down to 0.1 , respectively, with fixed $L a_{m}=0.05$. The velocity of the droplet increases as a result of decreasing the Laplace number. 
(a)

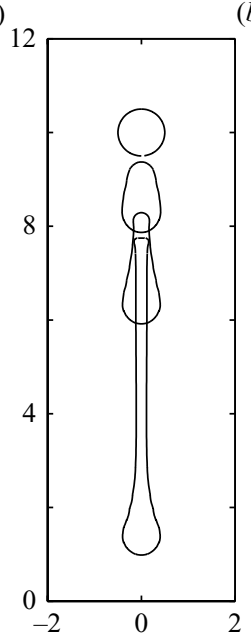

(b)

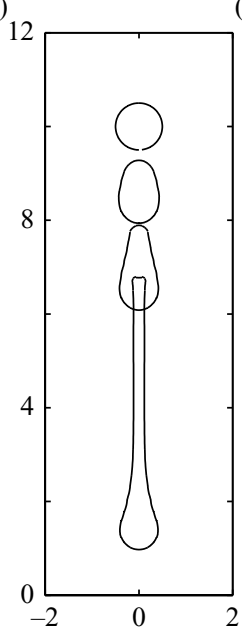

(c)

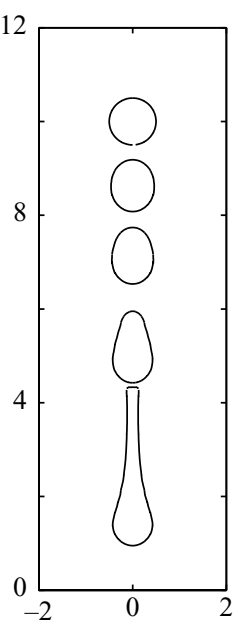

(d)

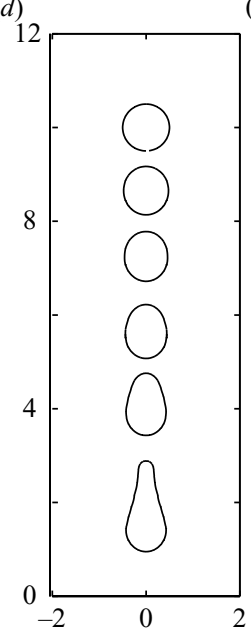

(e)

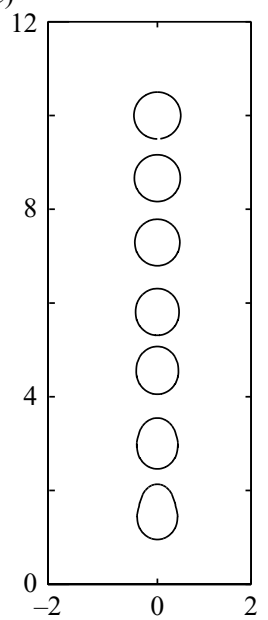

FIGURE 11. Droplet shapes at different Laplace numbers with fixed $L a_{m}=0.05$ : (a) $L a=0.002, \tau=0,75,112.5,131.9$, (b) $L a=0.01, \tau=0,75,112.5,133.2$, (c) $L a=0.04, \tau=$ $0,75,112.5,131.2,138.7, \quad(d) \quad L a=0.1, \quad \tau=0,75,112.5,131.2,138.7,142.5, \quad(e) \quad L a=0.4$, $\tau=0,75,112.5,131.2,138.7,142.5,145.2$. The magnetic Bond numbers are $B o_{m}=(a) 24,(b)$ $5,(c) 1,(d) 0.5,(e) 0.1$.

$\begin{array}{cccc}\begin{array}{c}\text { Droplet } \\ \text { diameter }(\mathrm{mm})\end{array} & \begin{array}{c}\text { Distance from } \\ \text { magnet }(\mathrm{mm})\end{array} & \begin{array}{c}\text { Simulation } \\ \text { time }(\mathrm{s})\end{array} & \begin{array}{c}\text { Experimental } \\ \text { time }(\mathrm{s})\end{array} \\ 1.0 & 12 & 960 & 900 \\ 1.8 & 12 & 270 & 240 \\ 2.0 & 11 & 170 & 150\end{array}$

TABLE 2. Comparison of numerical and experimental (Mefford et al. 2007) travel times for varying droplet sizes, initially at various distances from the magnet.

tension is estimated at $0.02 \mathrm{~N} \mathrm{~m}^{-1}$. The viscous medium with density of $998 \mathrm{~kg} \mathrm{~m}^{-3}$ and viscosity of $50 \mathrm{~Pa}$ s models the viscous humour in the eye.

We calculate the travel times for drop diameters $2 \mathrm{~mm}, 1.8 \mathrm{~mm}$ and $1 \mathrm{~mm}$, positioned at distances $11 \mathrm{~mm}, 12 \mathrm{~mm}$ and $12 \mathrm{~mm}$ away from the bottom of the domain, respectively. The magnetic Laplace number is of the order of $10^{-7}$, so that inertia is not important. Moreover, numerical results are checked to be independent of $L a_{m}$ in the asymptotic range $L a_{m} \ll 1$ even when $L a_{m}$ is taken to be of order 0.1. Since the time step required for accuracy in the numerical simulations is less restrictive for larger $L a_{m}$, an optimal value is found to minimize the total computational time. The parameter that influences drop shape is the ratio of magnetic force to interfacial tension force given by the magnetic Bond number, ranging from 0.03 to 0.06 . The experimental data of Mefford et al. (2007) is fitted with a Langevin function to describe the magnetization versus the magnetic field. For the first and second cases, the mesh size is set to $\Delta=0.1 \mathrm{~mm}$ and the time step is $\Delta t=0.001 \mathrm{~s}$. For the third case, the mesh size is set to $\Delta=0.0625 \mathrm{~mm}$ and the time step is $\Delta t=0.0005 \mathrm{~s}$. Table 2 shows that the computed travel times predict the experimentally observed values well. 
(a)

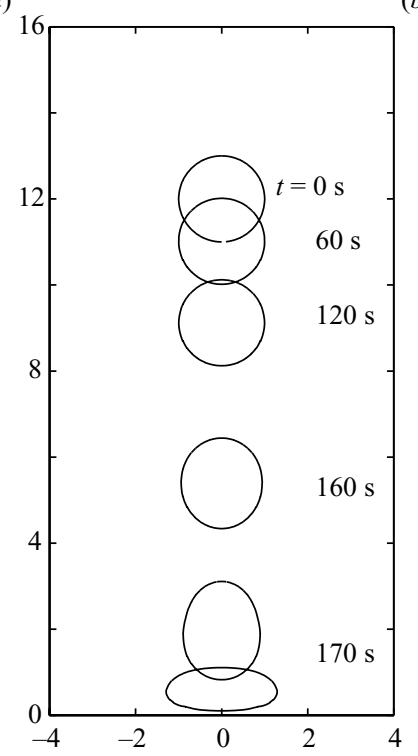

(b)

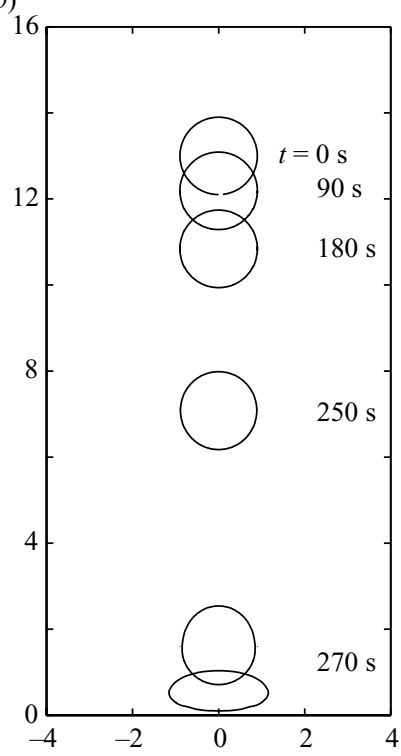

(c)

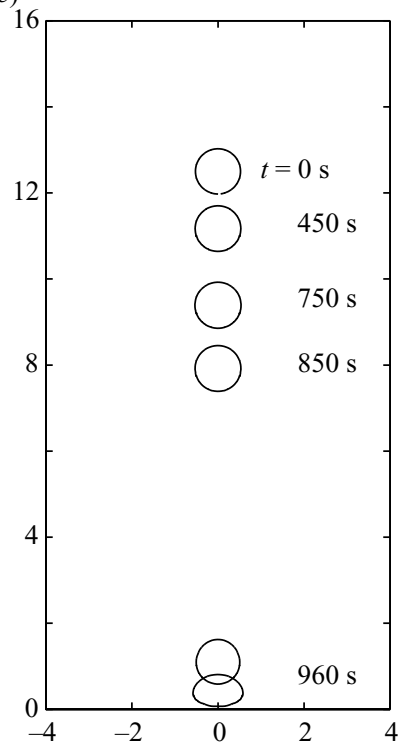

FIGURE 12. Droplet shapes for different diameter droplets positioned at various distances away from the bottom of the domain and driven by a magnetic field through a viscous medium with a viscosity of $50 \mathrm{Pas}$. (a) A $2 \mathrm{~mm}$ diameter droplet placed $11 \mathrm{~mm}$ away from the bottom of the domain, (b) a $1.8 \mathrm{~mm}$ droplet placed $12 \mathrm{~mm}$ away from the bottom of the domain, and (c) a $1 \mathrm{~mm}$ droplet placed $12 \mathrm{~mm}$ away from the bottom of the domain. The magnetic Bond numbers are $B o_{m}=(a) 0.063,(b) 0.056,(c) 0.03$.

Figure 12 shows the shape of the droplet as it travels through the viscous medium. As expected, higher velocities are computed for larger droplets and the travel time increases significantly for droplets placed further away from the magnet compared to a droplet placed closer. Also, a larger droplet deforms from a sphere to an oval as it approaches the magnet. This illustrates how the magnetic field gradient in the domain contributes to the deformation of the droplet, since the front of the droplet experiences a greater magnetic force in comparison to the back of the droplet. However, the shape of the smaller droplets remains nearly circular at all times except when they are about to hit the solid surface. Also, the deformation of the $1 \mathrm{~mm}$ diameter droplet (figure 12c) is less dramatic when it is close to hitting the target, which is due to higher surface tension effects and a decrease of the droplet velocity. These results are typical of the behaviour at low magnetic Bond numbers.

Figure 13 shows the velocity fields at times $t=120,160$ and $170 \mathrm{~s}$ for a droplet of $2 \mathrm{~mm}$ diameter placed $11 \mathrm{~mm}$ away from the bottom of the domain. This figure provides further details on the motion of the droplet. The velocity fields clearly demonstrate the following stages in drop evolution: at an early stage, the flow occurs approximately downwards and only in the region close to the droplet. A vortical flow in the viscous medium forms in front of the droplet. As the droplet approaches the magnet, these vortices induced in the viscous medium become stronger. When the droplet reaches the bottom of the domain, the vortices move towards the top of the droplet. At this stage, the flow inside the droplet is pumped outward from the centre of the droplet and results in the flattening of the droplet and consequently a decrease in the droplet height. 

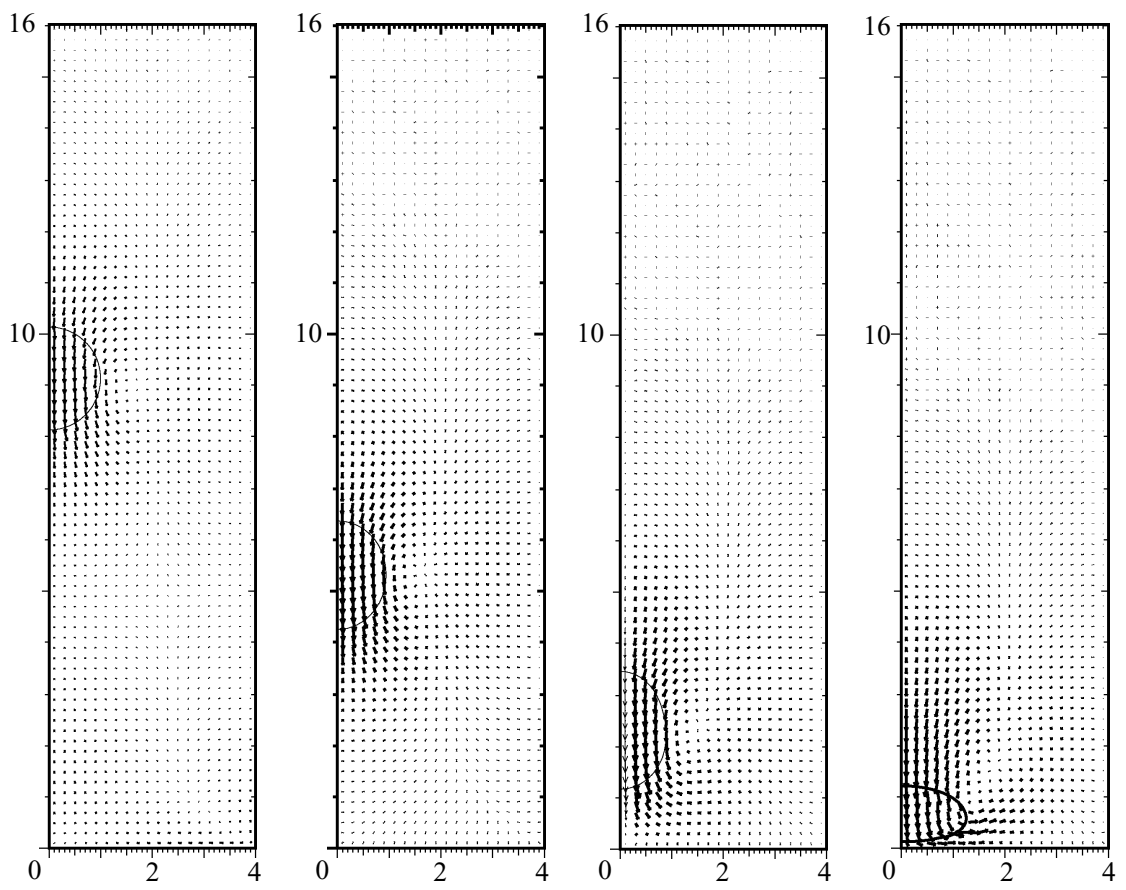

FIGURE 13. Velocity fields at times $t=120,160$ and $170 \mathrm{~s}$ (from left to right) for a droplet of $2 \mathrm{~mm}$ diameter placed $11 \mathrm{~mm}$ away from the bottom of the domain. $B o_{m}=0.06$.

\section{Conclusions}

We present a derivation for a model and numerical method to simulate the fluid motion coupled with the magnetic field and associated interfacial tension force in the flow of a ferrofluid droplet through a viscous medium under the influence of an externally applied magnetic field. The ferrohydrodynamic equations and a simple constitutive law are used to model the magnetic force acting at the interface. The numerical boundary condition for the simulation of the magnetic field is based upon measured values at the centreline of the domain in the absence of the drop. A conservative representation of the magnetic field force for immiscible two-fluid systems is derived.

The droplet undergoes dramatic deformation owing to the presence of an external magnetic field gradient. Its shape is influenced by the magnetic Bond number, as well as inertia. The simulations show that the droplet velocity is mainly influenced by the competition between the magnetic force which is proportional to the volume, and the viscous drag force which is proportional to the radius. Hence, the larger the drop, the faster the speed. The initial distance between the ferrofluid droplet and the external magnet is varied, and the simulated transit times agree well with the experimental measurements of Mefford et al. (2007). Our study shows that the deformation of the drop accounts for the difference in the transit times between prior models based on a spherical drop and the experimental data.

This research is supported by NSF-DMS 0405810, NCSA TG-CTS060013N and NSF-ARC Materials World Network for the Study of Macromolecular Ferrofluids (DMR-0602932 -LX0668968). We thank O. T. Mefford for discussions and data, and the referee who provided extensive comments for improvement. 


\section{Appendix}

The force acting on a magnetic particle of magnetic moment $\boldsymbol{m}$ is $\mu_{0}(\boldsymbol{m} \cdot \nabla) \boldsymbol{H}$ (Rosensweig 1985). For a magnetized body, this leads to a force density

$$
\boldsymbol{F}=\mu_{0}(\boldsymbol{M} \cdot \nabla) \boldsymbol{H} .
$$

This expression for the magnetic force, however, is not meaningful in the presence of an interface where both $\boldsymbol{M}$ and $\boldsymbol{H}$ have discontinuities. We therefore use the alternative form

$$
\boldsymbol{F}=\left(\left(\boldsymbol{B}-\mu_{0} \boldsymbol{H}\right) \cdot \nabla\right) \boldsymbol{H}=(\boldsymbol{B} \cdot \nabla) \boldsymbol{H}-\mu_{0}(\boldsymbol{H} \cdot \nabla) \boldsymbol{H} .
$$

Taking account of Maxwell's equations $\operatorname{div} \boldsymbol{B}=0$ and $\operatorname{curl} \boldsymbol{H}=\mathbf{0}$, we find

$$
\boldsymbol{F}=\operatorname{div}\left(\boldsymbol{H} \boldsymbol{B}^{T}\right)-\frac{1}{2} \mu_{0} \nabla|\boldsymbol{H}|^{2} .
$$

This conservative form remains meaningful in the presence of discontinuous interfaces. We now set $\boldsymbol{\sigma}_{m}=\boldsymbol{H} \boldsymbol{B}^{T}$. The second term, $-(1 / 2) \mu_{0} \nabla|\boldsymbol{H}|^{2}$ is proportional to the identity matrix, and is absorbed into the pressure field for the entire domain. This does not alter the interfacial force balance because it is implicitly consistent with the weak formulation which is discretized.

In axisymmetric coordinates the magnetic stress tensor is

$$
\boldsymbol{\sigma}_{m}=\mu\left[\begin{array}{ccc}
\left(\frac{\partial \phi}{\partial r}\right)^{2} & \frac{\partial \phi}{\partial r} \frac{\partial \phi}{\partial z} & 0 \\
\frac{\partial \phi}{\partial r} \frac{\partial \phi}{\partial z} & \left(\frac{\partial \phi}{\partial z}\right)^{2} & 0 \\
0 & 0 & 0
\end{array}\right],
$$

and

$$
\nabla \cdot \boldsymbol{\sigma}_{m}=\left[\frac{1}{r} \frac{\partial}{\partial r}\left[r\left(\boldsymbol{\sigma}_{m}\right)_{r r}\right]+\frac{\partial}{\partial z}\left[\left(\boldsymbol{\sigma}_{m}\right)_{r z}\right]\right] \boldsymbol{e}_{\boldsymbol{r}}+\left[\frac{1}{r} \frac{\partial}{\partial r}\left[r\left(\boldsymbol{\sigma}_{m}\right)_{r z}\right]+\frac{\partial}{\partial z}\left[\left(\boldsymbol{\sigma}_{m}\right)_{z z}\right]\right] \boldsymbol{e}_{z},
$$

where $\boldsymbol{e}_{\boldsymbol{r}}$ and $\boldsymbol{e}_{z}$ are unit vectors in $r$ and $z$ directions.

In the momentum equation (2.12), the rate of deformation tensor in axisymmetric coordinates is

and

$$
\boldsymbol{S}_{i, j}=\left[\begin{array}{ccc}
2 \frac{\partial v_{r}}{\partial r} & \frac{\partial v_{r}}{\partial z}+\frac{\partial v_{z}}{\partial r} & 0 \\
\frac{\partial v_{r}}{\partial z}+\frac{\partial v_{z}}{\partial r} & 2 \frac{\partial v_{z}}{\partial z} & 0 \\
0 & 0 & 2 \frac{v_{r}}{r}
\end{array}\right]
$$

$$
\nabla \cdot \eta \boldsymbol{S}=\left[\frac{1}{r} \frac{\partial}{\partial r}\left(r \eta S_{r r}\right)+\frac{\partial}{\partial z} \eta S_{r z}-\frac{1}{r} \eta S_{\theta \theta}\right] \boldsymbol{e}_{r}+\left[\frac{1}{r} \frac{\partial}{\partial r}\left(r \eta S_{r z}\right)+\frac{\partial}{\partial z} \eta S_{z z}\right] \boldsymbol{e}_{z} .
$$

\section{REFERENCES}

BACRI, J. C. \& SALIN, D. 1982 Instability of ferrofluid magnetic drops under magnetic field. J. Phys. Lett. 43, 649-654.

Bashtovoi, V., Lavrova, O. A., Polevikov, V. K. \& Tobiska, L. 2002 Computer modeling of the instability of a horizontal magnetic-fluid layer in a uniform magnetic field. J. Magn. Magn. Mater. 252, 299-301. 
Baygents, J. C., Rivette, N. J. \& Stone, H. A. 1998 Electrohydrodynamic deformation and interaction of drop pairs. J. Fluid Mech. 368, 359-375.

Brackbill, J. U., Kothe, D. B. \& Zemach, C. 1992 A continuum method for modeling surface tension. J. Comput. Phys. 100, 335-354.

Clift, R., Grace, J. R. \& Weber, M. E. 1978 Bubbles, Drops, and Particles. Academic.

Knieling, H., Richter, R., Rehberg, I., Matthies, G. \& Lange, A. 2007 Growth of surface undulations at the Rosensweig instability. Phys. Rev. E 76, 066301.

Lafaurie, B., Nardone, C., Scardovelli, R., Zaleski, S. \& Zanetti, G. 1994 Modelling merging and fragmentation in multiphase flows with SURFER. J. Comput. Phys. 113, 134-147.

Lavrova, O., Matthies, G., Polevikov, V. \& Tobiska, L. 2004 Numerical modeling of the equilibrium shapes of a ferrofluid drop in an external magnetic field. Proc. Appl. Math. Mech. 4, 704-705.

Lavrova, O., Matthies, G., Mitkova, T., Polevikov, V. \& Tobiska, L. 2006 Numerical treatment of free surface problems in ferrohydrodynamics. J. Phys.: Condens. Matter 18, S2657-S2669.

LI, J. \& RENARDY, Y. 1999 Direct simulation of unsteady axisymmetric core-annular flow with high viscosity ratio. J. Fluid Mech. 391, 123-149.

Li, J., Renardy, Y. \& Renardy, M. 2000 Numerical simulation of breakup of a viscous drop in simple shear flow through a volume-of-fluid method. Phys. Fluids 12, 269-282.

Liu, X., Kaminski, M. D., Riffle, J. S., Chen, H., Torno, M., Finck, M. R., Taylor, L. \& Rosengart, A. J. 2007 Preparation and characterization of biodegradable magnetic carriers by single emulsion-solvent evaporation. J. Magn. Magn. Mat. 311, 84-87.

Matthies, G. \& Tobiska, L. 2005 Numerical simulation of normal-field instability in the static and dynamic case. J. Magn. Magn. Mater. 289, 346-349.

Mefford, O. T., Woodward, R. C., Goff, J. D., Vadala, T. P., St Pierre, T. G., Dailey, J. P. \& RiffLE, J. S. 2007 Field-induced motion of ferrofluids through immiscible viscous media: testbed for restorative treatment of retinal detachment. J. Magn. Magn. Mater. 311, 347-353.

Nickerson, C. S., Karageozian, H. L., Park, J. J. \& Kornfield, J. A. 2005 Internal tension: a novel hypothesis concerning the mechanical properties of the vitreous humor. Macromol. Symp. 227, 183-189.

Patankar, S. V. 1980 Numerical Heat Transfer and Fluid Flow. McGraw-Hill.

Rosensweig, R. E. 1985 Ferrohydrodynamics. Cambridge University Press.

SCARdovelli, R. \& ZALESKi, S. 1999 Direct numerical simulation of free surface and interfacial flow. Annu. Rev. Fluid Mech. 31, 567-604.

Voltairas, P. A., Fotiadis, D. I. \& Michalis, L. K. 2002 Hydrodynamics of magnetic drug targeting. J. Biomech. 35, 813-821. 